\title{
Susceptibility to the Sugar Beet Cyst Nematode Is Modulated by Ethylene Signal Transduction in Arabidopsis thaliana
}

\author{
Martin J. E. Wubben II, ${ }^{1}$ Hong Su, ${ }^{1}$ Steven R. Rodermel, ${ }^{1,2}$ and Thomas J. Baum ${ }^{1,3}$ \\ ${ }^{1}$ Interdepartmental Genetics Program, ${ }^{2}$ Department of Botany, and ${ }^{3}$ Department of Plant Pathology, lowa \\ State University, Ames 50011, U.S.A. \\ Submitted 14 March 2001; Accepted 15 June 2001.
}

Previously, we identified Arabidopsis thaliana mutant rhd1-4 as hypersusceptible to the sugar beet cyst nematode Heterodera schachtii. We assessed rhd1-4 as well as two other rhdl alleles and found that each exhibited, in addition to $H$. schachtii hypersusceptibility, decreased root length, increased root hair length and density, and deformation of the root epidermal cells compared with wildtype A. thaliana ecotype Columbia (Col-0). Treatment of rhd1-4 and Col-0 with the ethylene inhibitors 2-aminoethoxyvinylglycine and silver nitrate and the ethylene precursor 1-aminocyclopropane-1-carboxylic acid suggests that the rhd1-4 hypersusceptibility and root morphology phenotypes are the result of an increased ethylene response. Assessment of known ethylene mutants further support the finding that ethylene plays a role in mediating $A$. thaliana susceptibility to $H$. schachtii because mutants that overproduce ethylene (eto1-1, eto2, and eto3) are hypersusceptible to $H$. schachtii and mutants that are ethylene-insensitive (etr1-1, ein2-1, ein3-1, eir1-1, and axr2) are less susceptible to $H$. schachtii. Because the ethylene mutants tested show altered susceptibility and altered root hair density and length, a discrimination between the effects of altered ethylene signal transduction and root hair density on susceptibility was accomplished by analyzing the $t$ tg and $g l 2$ mutants, which produce ectopic root hairs that result in greatly increased root hair densities while maintaining normal ethylene signal transduction. The observed normal susceptibilities to $H$. schachtii of $t$ tg and $g l 2$ indicate that increased root hair density, per se, does not cause hypersusceptibility. Furthermore, the results of nematode attraction assays suggest that the hypersusceptibility of rhd1-4 and the ethylene-overproducing mutant eto3 may be the result of increased attraction of $H$. schachtii-infective juveniles to root exudates of these plants. Our findings indicate that rhd1 is altered in its ethylene response and that ethylene signal transduction positively influences plant susceptibility to cyst nematodes.

Cyst nematodes of the genus Heterodera are obligate, sedentary endoparasites of many important agricultural crops.

Corresponding author: T. J. Baum; Fax: +1 515-294-9420; E-mail: tbaum@iastate.edu
These nematodes have developed highly evolved relationships with their respective hosts. In a typical Heterodera spp. life cycle, an infective second-stage juvenile (J2) hatches from an egg in the soil and is attracted to the elongation zone of the host root (Wyss and Zunke 1986; Wyss and Grundler 1992). Epidermal penetration and intracellular migration through the root cortex are facilitated by cell wall-degrading enzymes secreted by the nematode (De Boer et al. 1999; Smant et al. 1998; Wang et al. 1999). The J2 then selects an initial feeding cell in the vascular cylinder and pierces it with its stylet, a hollow, protrusible mouth spear (Wyss and Grundler 1992). The initial feeding cell undergoes significant physiological and morphological changes that culminate in the fusion of the initial feeding cell with neighboring cells by partial cell wall dissolution, forming an expanding syncytium (Jones 1981; Wyss 1992; Wyss and Grundler 1992). The syncytium provides the sedentary nematode with the nutrients needed to develop through the third-stage juvenile (J3) and fourth-stage juvenile (J4) into a reproductive male or female adult. Microscopic observations revealed that nematode secretions are injected through the stylet into or around the initial feeding cell where they may act as molecular signals triggering unknown signal transduction mechanisms that cause syncytium formation and maintenance (Davis et al. 2000).

The successful completion of the Heterodera life cycle constitutes a compatible interaction. Exploration of a compatible cyst nematode-plant interaction with mutant plants altered in their susceptibility to cyst nematodes bears promise to elucidate the molecular events of nematode-plant signal exchange as well as to identify the plant genes necessary for successful Heterodera parasitism. Previously, we developed an in vitro mutant screening procedure and identified Arabidopsis thaliana mutants exhibiting altered susceptibility to the sugar beet cyst nematode Heterodera schachtii (Baum et al. 2000). The hypersusceptible mutant line 2-4-6 was identified, and its mutant gene was determined to be allelic to rhdl (root hair defective) (Schiefelbein and Somerville 1990) and reb1-1 and rebl-2 (root epidermal bulger) (Baskin et al. 1992). Because it was the fourth-known rhdl allele, we named the mutant gene in line 2-4-6 rhdl-4 (Baum et al. 2000). Here, we present an analysis of the physiological nature of the rhdl-4 phenotypes, which led to the assessment of ethylene signal transduction as it pertains to cyst nematode parasitism. 


\section{RESULTS}

All of the rhdl alleles evaluated, i.e., rhdl, rebl-1, and rhd1-4 (rebl-2 was not assessed as a result of its temperaturesensitive nature) (Baskin et al. 1992), are hypersusceptible to $H$. schachtii and are morphologically indistinguishable (Baum et al. 2000). This hypersusceptibility is manifested as an approximately twofold increase in the number of $\mathrm{J} 4$ females able to develop compared with wild-type $A$. thaliana cv. Columbia (Col-0) (Baum et al. 2000). The rhdl-4 hypersusceptibility is especially interesting because the root of this mutant is significantly shorter than normal, resulting in less root tissue for the nematode to infect. In addition, rhdl-4 develops more and longer root hairs relative to Col-0 (Table 1 and Fig. 1A and B), and a portion of the rhdl-4 root epidermal cells are deformed and exhibit a bulging phenotype (Fig. 1C). All rhdl-4 phenotypes can be attributed to a single recessive allele (Baum et al. 2000). We did not observe any alterations in the development or morphology of shoot tissues in light-grown or etiolated rhd1-4 seedlings, suggesting that the rhdl mutation has a rootspecific effect (data not shown).

Modulation of rhd1-4 ethylene production and perception.

The shortened roots, increased root hair length, and increased root hair density phenotypes of rhd1-4 were reminiscent of the phenotypes of wild-type $A$. thaliana seedlings grown in the presence of excess ethylene (Eliasson and Bollmark 1988; Pitts et al. 1998; Tanimoto et al. 1995). To evaluate the possible role of ethylene in mediating the rhdl-4 phenotypes, we examined the effects of two ethylene inhibitors and an ethylene precursor on rhd1-4 and Col-0 root morphology, root hair development, and susceptibility to $H$. schachtii.

\section{2-Aminoethoxyvinylglycine (AVG).}

In planta ethylene production is inhibited by AVG, which inhibits the enzyme 1-aminocyclopropane-1-carboxylic acid (ACC) synthase, slowing the conversion of S-adenosylmethionine to the ethylene precursor ACC (Yang and Hoffman 1984). AVG concentrations as low as $0.5 \mu \mathrm{M}$ significantly inhibit root hair initiation in A. thaliana (Tanimoto et al. 1995). rhd1-4 and Col-0 seedlings were germinated and grown on nutrient media containing AVG at concentrations of 0.05 to $2.5 \mu \mathrm{M}$. Root hair lengths and densities decreased in rhdl-4 and Col-0 as AVG concentrations increased (data not shown). AVG $(1.0 \mu \mathrm{M})$ restored $r h d 1-4$ root hair length and density to approximately normal levels and completely suppressed the root epidermal cell deformation phenotype (Fig. 1D). Furthermore, AVG-treated rhd1-4 and Col-0 plants were less susceptible to $H$. schachtii, with both genotypes supporting fewer sedentary $\mathrm{J} 2$ at 7 days postinoculation (dpi) and fewer J4 females at 15 dpi compared with plants grown on medium lack-

Table 1. Root and root hair measurements of wild-type Arabidopsis thaliana ecotype Columbia (Col-0) and rhd1-4

\begin{tabular}{lccc}
\hline Strains & $\begin{array}{c}\text { Root } \\
\text { length }(\mathbf{m m})^{\mathbf{a}}\end{array}$ & $\begin{array}{c}\text { Root hair } \\
\text { length }(\mathbf{m m})^{\mathbf{a}}\end{array}$ & $\begin{array}{c}\text { Root hair } \\
\text { density }(\mathbf{n o .} / \mathbf{m m})^{\mathbf{a}}\end{array}$ \\
\hline Col-0 & $53 \pm 2$ & $0.28 \pm 0.02$ & $21.2 \pm 1.1$ \\
rhd1-4 & $31 \pm 1$ & $0.59 \pm 0.03$ & $27.9 \pm 2.2$ \\
\hline
\end{tabular}

${ }^{\text {a }}$ Root length, root hair length, and root hair density of rhd1-4 are significantly different from Col-0 as determined by paired Student's $t$ test $(P<0.05)$. The mean and standard error of the mean are presented. ing AVG (Fig. 2). The observation that AVG, an inhibitor of ethylene production, restored all rhd1-4 phenotypes to normal suggests that the rhdl-4 phenotypes are the result of an increased ethylene response.

\section{$\mathrm{AgNO}_{3}$.}

A. thaliana ethylene perception is inhibited by silver ions $\left(\mathrm{Ag}^{+}\right)$that inactivate at least one of the ethylene receptors

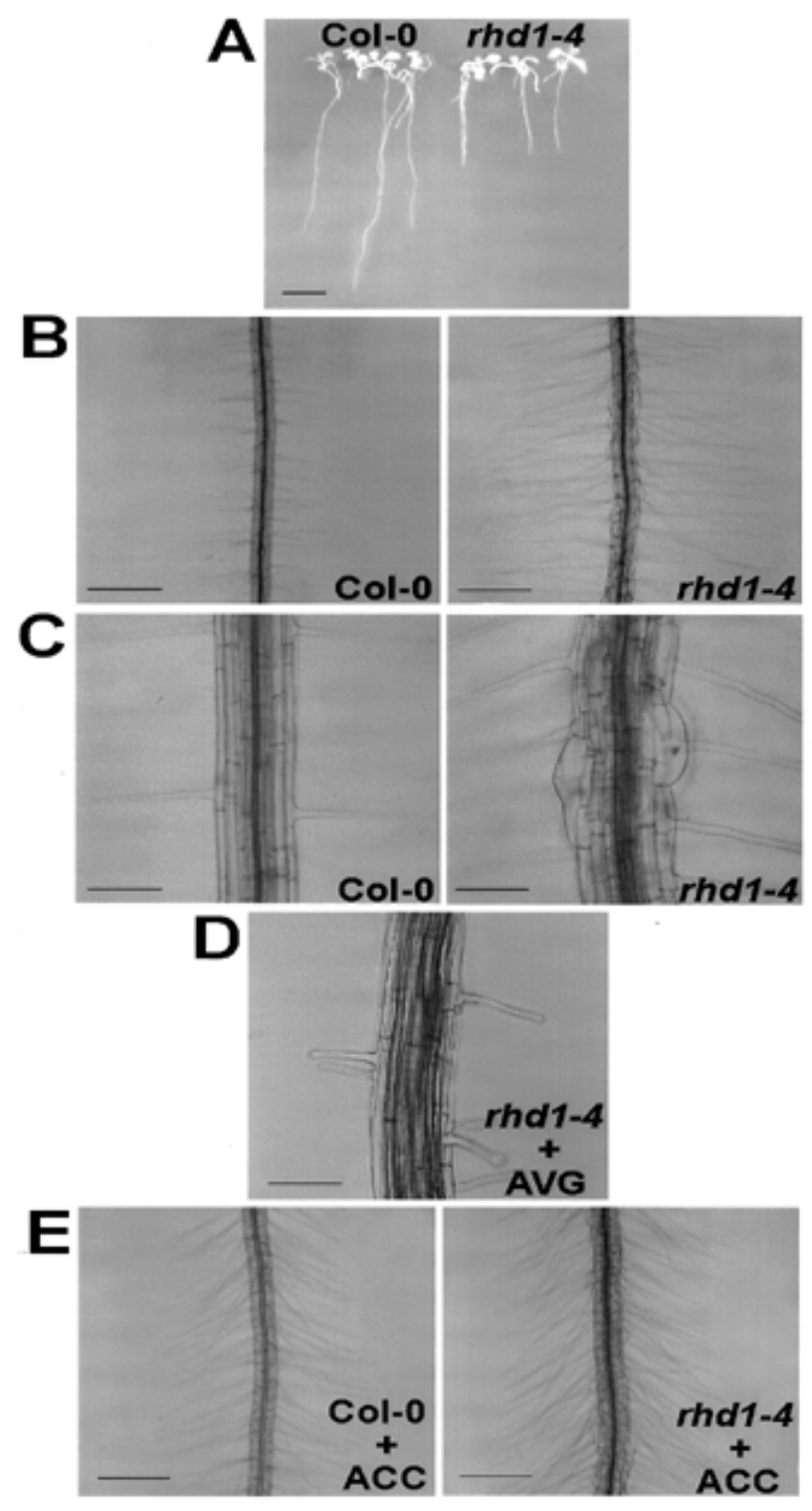

Fig. 1. Comparison of root length, root hair development, and root epidermal cell morphology of wild-type Arabidopsis thaliana ecotype Columbia (Col-0) and A. thaliana mutant rhd1-4 plants grown on normal medium and 2-aminoethoxyvinylglycine (AVG)- or 1-aminocyclo-propane-1-carboxylic acid (ACC)-supplemented medium. A, Macroscopic comparison of Col-0 and rhd1-4. Scale bar $=1,000 \mu \mathrm{m}$. B, Comparison of Col-0 and rhd1-4 root hair phenotypes. Scale bar $=400 \mu \mathrm{m}$. C, Comparison of root epidermal cell morphology of Col-0 and rhd1-4. Scale bar $=100 \mu \mathrm{m}$. D, Effect of $1.0 \mu \mathrm{M} \mathrm{AVG}$ on rhd1-4 root epidermis and root hairs (compare to $\mathbf{C}$ ). Scale bar $=100 \mu \mathrm{m}$. E, Effects of the ethylene precursor ACC on root hair development in Col-0 and rhd1-4 (compare to B). Scale bars $=400 \mu \mathrm{m}$. 
(ETR1) (Beyer 1976; Beyer 1979; Rodriguez et al. 1999). A. thaliana root hair initiation is inhibited by $1.0 \mu \mathrm{M} \mathrm{\textrm {Ag } ^ { + }}$ (Tanimoto et al. 1995). We found that $\mathrm{Ag}^{+}$concentrations of 1.0 to $10.0 \mu \mathrm{M}$ progressively reduced root hair lengths and root hair densities of rhdl-4 and Col-0 seedlings growing in nutrient medium (data not shown). These observations are consistent with the idea that $r h d 1-4$ exhibits an increased ethylene response. In contrast to the AVG data, $\mathrm{Ag}^{+}$did not suppress the root epidermal cell deformation phenotype in rhdl-4. Attempts were made to determine the susceptibility of $\mathrm{Ag}^{+}-$ treated plants to $H$. schachtii. As a result of the strong effects of $\mathrm{Ag}^{+}$on general root morphology, however, a meaningful susceptibility analysis could not be conducted.

\section{ACC.}

Increasing the availability of the ethylene precursor ACC elevates endogenous ethylene concentrations in plants (Yang and Hoffman 1984). ACC $(1.0 \mu \mathrm{M})$ promotes root hair development in A. thaliana (Tanimoto et al. 1995), and increased ethylene concentrations inhibit root elongation (Eliasson and Bollmark 1988). If the rhdl-4 phenotypes are the result of an increased ethylene response, as suggested by the AVG and $\mathrm{Ag}^{+}$data, then the rhdl-4 phenotypes should be phenocopied in Col-0 plants supplemented with ACC. In support of this notion, we observed that ACC concentrations of 0.05 to $0.5 \mu \mathrm{M}$ caused Col-0 seedlings to produce more and longer root hairs (Fig. 1E; compare to untreated rhdl-4 in Fig. 1B) while simultaneously producing shorter root lengths (data not shown).
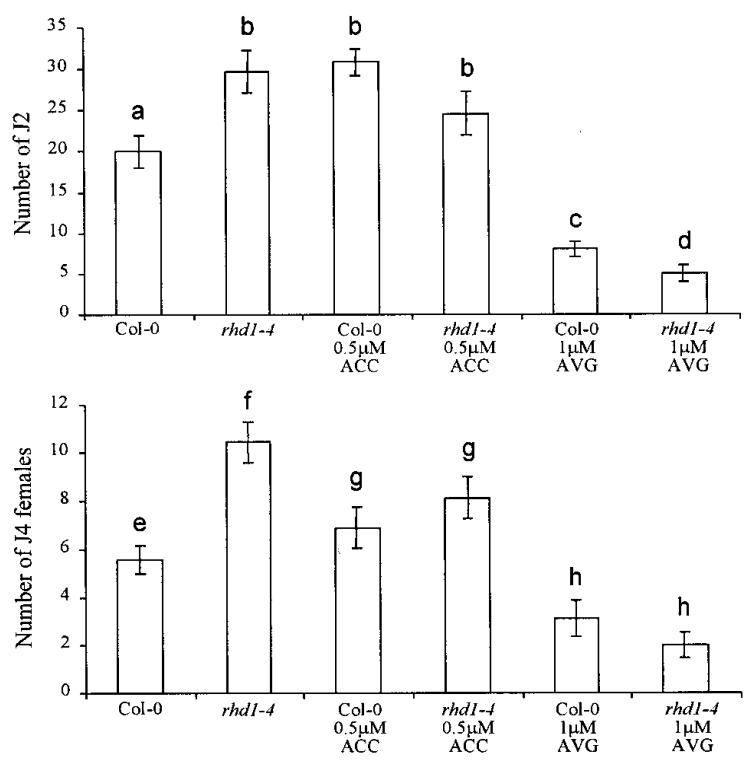

Fig. 2. Effects of an ethylene inhibitor 2-aminoethoxyvinylglycine (AVG) and ethylene precursor 1-aminocyclopropane-1-carboxylic acid (ACC) on nematode susceptibility in wild-type Arabidopsis thaliana ecotype Columbia (Col-0) and A. thaliana mutant rhd1-4. Susceptibility was determined by counting sedentary second-stage juvenile nematodes (J2) at 7 days postinoculation (dpi) and counting female fourth-stage juvenile nematodes (J4) at $15 \mathrm{dpi}$. Col-0 and rhd1-4 control plants received appropriate amounts of sterile distilled water instead of AVG or ACC solution. Means and standard error of the means are shown. Significance was determined by paired Student's $t$ test $(P<0.05)$. Means sharing the same letter designation are not significantly different.
The application of ACC also caused rhdl-4 to produce more root hairs (Fig. 1E) and further decreased the rhdl-4 root length (data not shown). Additionally, $0.5 \mu \mathrm{M}$ ACC caused an increase in $\mathrm{H}$. schachtii $\mathrm{J} 2$ and $\mathrm{J} 4$ female numbers on Col-0 at 7 and $15 \mathrm{dpi}$, respectively (Fig. 2). The susceptibility of ACCtreated $r h d 1-4$ plants was not increased, possibly indicating a saturation of the ethylene effect on nematode susceptibility. The observed decrease in root length, increase in root hair length and density, and hypersusceptibility in ACC-treated Col-0 plants further support the notion that an increased ethylene response is responsible for the rhdl-4 phenotypes, with the exception that ACC-treated rhdl-4 plants did not show an increase in susceptibility.

The root epidermal cell morphology of rhdl-4 and Col-0 seedlings was not affected by the ACC concentrations used in this study, which indicates that the root epidermal cell deformation phenotype appears ethylene independent and, at most, only plays a minor role in mediating rhdl-4 hypersusceptibility to $H$. schachtii because ACC-treated Col-0 plants, which lack the root epidermal cell deformation phenotype, are hypersusceptible. More importantly, observing decreased susceptibility to $H$. schachtii of AVG-treated Col-0 plants and increased susceptibility of ACC-treated Col-0 plants in these studies strongly implicates ethylene in modulating plant susceptibility to this nematode.

\section{Susceptibility of $A$. thaliana ethylene and epidermal cell fate mutants.}

The influence of ethylene on plant susceptibility to $H$. schachtii was further investigated by examining the susceptibilities of $A$. thaliana mutants that either overproduce ethylene or are ethylene insensitive (Table 2). The ethylene-mediated changes in root hair density and length in these mutants were classified visually as increased, decreased, or normal, relative to Col-0 (Table 2). The root hair phenotypes of most of these mutants have been evaluated previously and are in agreement with our observations (Masucci and Schiefelbein 1996; Pitts et al. 1998; Schneider et al. 1997).

A positive correlation between root hair development and susceptibility to $H$. schachtii was apparent upon assessment of the ethylene mutants (Table 2). The ethylene-overproducing mutants eto1-1, eto2, and eto3, each having more and longer root hairs, consistently support more J4 females at $15 \mathrm{dpi}$, relative to Col-0. This observation is even more remarkable considering that eto mutants produce root systems that are approximately one-third to one-half the size of Col-0 plants (data not shown). In addition, attempts were made to determine the susceptibility of the constitutive triple response (ctr1-1) mutant (Guzman and Ecker 1990). ctrl-1 shows an increased ethylene response that is more pronounced than the ethylene response of the eto mutants, resulting in the development of a very small root system. This root system was too small to support nematode development, although large numbers of $\mathrm{J} 2$ were attracted to it (data not shown). Conversely, the ethylene-insensitive mutants etr 1-1, ein2-1, ein3-1, eir1-1, and $\operatorname{axr} 2$, which have decreased root hair lengths and/or densities, consistently hosted fewer J4 females at 15 dpi (Table 2). ein2, which exhibits the highest level of ethylene insensitivity, always showed the greatest reduction in J4 female numbers. Weaker ethylene-insensitive mutants (ein5, ein6, and ein7), i.e., those with little or no effects on root hair development, 
also were assessed for their susceptibility to $H$. schachtii. The susceptibilities of these "weak" mutants, however, varied considerably among experiments such that they did not show a clear susceptibility phenotype.

Throughout our experiments, a strong positive correlation between root hair density and/or length and susceptibility to $H$. schachtii was apparent. It was not clear, however, whether root hair alterations and susceptibility changes were independent pleiotropic effects of the rhdl-4 mutation or whether susceptibility to the nematode was, in fact, conditioned by increased root hair density and length. To determine whether increased root hair density alone would elevate plant susceptibility, we assessed $A$. thaliana mutants altered in root epidermal cell fate. In the $t t g$ and $g l 2$ mutants, virtually all root epidermal cells are fated to become hair cells, which greatly increases root hair density in the absence of altered ethylene production or signal transduction. This ectopic root hair formation is hypothesized to be the result of a lack of negative regulation of hair cell formation during epidermal cell fate determination (Masucci and Schiefelbein 1996). The observed wild-type level susceptibilities of $t \mathrm{tg}$ and $g l 2$ indicate that root hairs do not directly influence plant susceptibility to $H$. schachtii (Fig. 3). Therefore, the altered susceptibilities observed for $r h d l-4$ and the ethylene mutants are root hair independent but appear highly dependent on ethylene signal-transduction because ethylene-mediated increases in root hair numbers and lengths always were associated with increased susceptibilities to $H$. schachtii. Similarly, the opposite relationship held true.

\section{Attraction assays.}

Casual observations suggested that infective $H$. schachtii juveniles found the roots of rhd1-4, ctrl-1, and eto mutant plants as well as ACC-treated Col-0 plants in a shorter period of time than those of Col-0 plants grown on normal medium. These findings suggest that the hypersusceptibility of rhdl-4 may be the result of an increased attraction of infective juveniles to its roots, resulting in an increased number of sedentary J2 at 7 dpi (Fig. 2). To directly test this hypothesis, we used root exudates from rhd1-4, eto3, and Col-0 plants grown in vi- tro to conduct $\mathrm{J} 2$ attraction assays. Growth medium cores from the immediate vicinity of the plant roots were transferred to new medium plates and placed in pairwise combinations of two cores per plate. The following combinations of root exudates were tested: Col-0 versus a control lacking root exudates, rhd1-4 versus Col-0, and eto3 versus Col-0. Nematodes were placed in the center between cores, and the attraction of J2 into the cores was assayed over time. Twelve replications of each comparison were performed. Results (Table 3) indicate that eto 3 and rhdl-4 root exudates attracted more nematodes than exudates from Col-0 roots, whereas root exudates

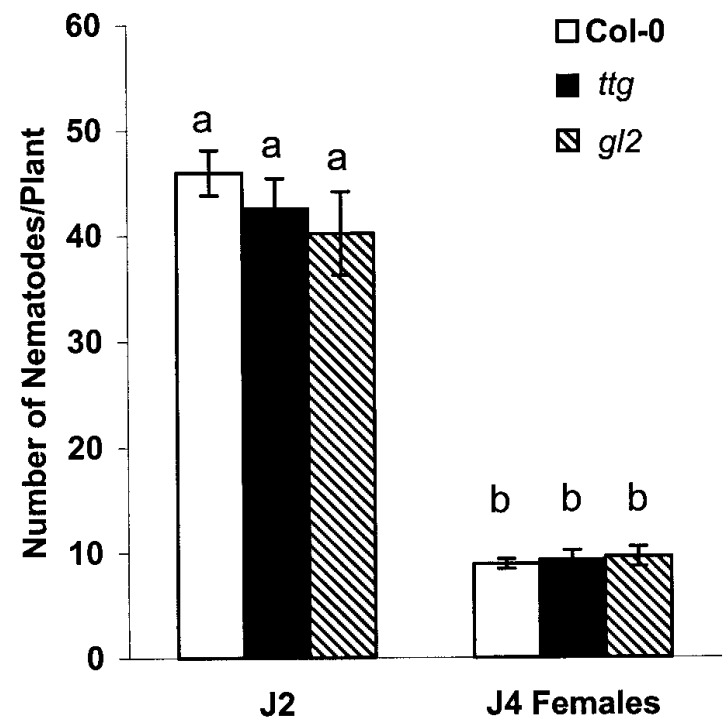

Fig. 3. Susceptibility of the $t t g$ and $g l 2$ epidermal cell fate mutants compared with wild-type Arabidopsis thaliana ecotype Columbia (Col-0). Sedentary second-stage juveniles (J2) and female fourth-stage juveniles (J4) were counted at 7 and 15 days postinoculation, respectively. Means and standard error of the means are shown. Significance was determined by paired Student's $t$ test $(P<0.05)$. Means sharing the same letter designation are not significantly different.

Table 2. Susceptibility of Arabidopsis thaliana ethylene mutants to Heterodera schachtii as determined by the total number of fourth-stage juvenile (J4) females at 15 days postinoculation (dpi)

\begin{tabular}{|c|c|c|c|c|c|c|}
\hline \multirow[b]{2}{*}{ Strains } & \multirow[b]{2}{*}{ Description } & \multirow[b]{2}{*}{ Root hair length } & \multirow[b]{2}{*}{ Root hair density } & \multicolumn{2}{|c|}{$\mathrm{J} 4$ females at $15 \mathrm{dpi}^{\mathrm{a}}$} & \multirow[b]{2}{*}{ Reference } \\
\hline & & & & Experiments 1 and 2 & Experiment 3 & \\
\hline Col-0 & Wild-type strain & & & $6.4 \pm 0.7$ & $6.0 \pm 0.7^{b}$ & \\
\hline rhd $1-4$ & Root hair defective & Increased & Increased & $9.3 \pm 0.7^{c}$ & $9.6 \pm 1.0^{c}$ & \\
\hline etol-1 & Ethylene overproducer & Increased & Increased & $13.6 \pm 0.9^{c}$ & $7.1 \pm 0.6$ & Guzman and Ecker 1990 \\
\hline eto 2 & Ethylene overproducer & Increased & Increased & $11.3 \pm 1.6^{\mathrm{c}}$ & $7.2 \pm 1.0$ & $\begin{array}{l}\text { Kieber and Ecker 1993; } \\
\text { Kieber et al. } 1993\end{array}$ \\
\hline eto3 & Ethylene overproducer & Increased & Increased & $12.7 \pm 1.3^{\mathrm{c}}$ & $7.5 \pm 1.0$ & Kieber and Ecker 1993 \\
\hline etrl-1 & Ethylene receptor & Decreased & Normal & $3.0 \pm 0.7^{\mathrm{c}}$ & $2.8 \pm 0.7^{\mathrm{c}}$ & Bleecker et al. 1988 \\
\hline ein2-1 & Ethylene insensitive & Decreased & Normal & $1.4 \pm 0.8^{\mathrm{c}}$ & $1.4 \pm 0.6^{\mathrm{c}}$ & Guzman and Ecker 1990 \\
\hline ein3-1 & Ethylene insensitive & Decreased & Normal & $3.9 \pm 0.8^{c}$ & Not determined & $\begin{array}{l}\text { Kieber et al. 1993; } \\
\text { Roman et al. } 1995\end{array}$ \\
\hline eirl-1 & Ethylene insensitive root & Decreased & Not determined & $3.5 \pm 0.9^{c}$ & $1.5 \pm 0.5^{\mathrm{c}}$ & Roman et al. 1995 \\
\hline axr2 & Auxin resistant & Not determined & Decreased & $4.2 \pm 0.8^{c}$ & $3.0 \pm 0.6^{\mathrm{c}}$ & Wilson et al. 1990 \\
\hline
\end{tabular}

${ }^{a}$ Data from experiments 1 and 2 were combined and analyzed. Means and standard errors of the means are shown as determined by least square means analysis.

${ }^{\mathrm{b}}$ Mutant strains assessed in experiment 3 were divided into three separate inoculation events to increase the number of observations for each mutant, therefore, each inoculation event had its own wild-type mean. The presented wild-type mean is from one such inoculation event.

${ }^{\mathrm{c}}$ Significantly different from wild-type Arabidopsis thaliana ecotype Columbia (Col-0) as determined by least significant difference analysis (two tailed; $P<0.05$ ). 
from Col- 0 were more attractive than the control. These findings suggest that an increased attraction of $\mathrm{J} 2$ to the roots of rhd1-4 and eto3 may contribute to their hypersusceptibility.

\section{DISCUSSION}

We reported previously on a fourth rhdl mutant allele, termed rhd1-4 (Baum et al. 2000). In this article, we show that, in addition to hypersusceptibility to $H$. schachtii, plants with the rhd1-4 allele exhibit shorter roots, increased root hair density and length, and a deformation of the root epidermal cells. In addition, the absence of any alteration in the development and morphology of rhdl-4 shoot tissues suggests that the effects of the rhdl mutation are root specific.

Shorter roots and increased root hair density and length are hallmarks of plant development mediated by elevated ethylene concentrations (Cao et al. 1999; Eliasson and Bollmark 1988; Tanimoto et al. 1995). The modulation of rhdl-4 and Col-0 ethylene production (via AVG and ACC) and ethylene perception (via $\mathrm{AgNO}_{3}$ ) strongly indicate an increased ethylene response as a cause for the rhdl-4 phenotypes. The fact that AVG, an inhibitor of ethylene production, could rescue all rhdl-4 phenotypes indicates that the rhdl-4 phenotypes are not the result of constitutive activation of the ethylene signal transduction pathway because phenotypes of constitutive ethylene response mutants such as ctrl are not affected by the application of inhibitors of ethylene production like AVG (Guzman and Ecker 1990).

The inoculation of known ethylene mutants with altered root hair phenotypes resulting from ethylene overproduction or ethylene insensitivity also revealed a positive correlation between ethylene effects and susceptibility to the nematode (Table 2). These results are in agreement with the susceptibilities recorded for ACC- and AVG-treated Col-0 and rhdl-4 plants. In general, the overproduction of ethylene resulting from mutation or ACC treatment results in hypersusceptibility. By the same token, ethylene insensitivity resulting from mutation or from inhibition of ethylene production (via AVG) decreases susceptibility. We observed, however, that altered root hair development, i.e., increased root hair density and length upon ethylene overproduction or ACC treatment and decreased root hair density and length resulting from ethylene insensitivity or AVG treatment, also correlated with susceptibility changes. Interestingly, assessment of the $t t g$ and $g l 2 \mathrm{mu}-$ tants, which have large numbers of ectopic root hairs but are not perturbed in either ethylene production or signal transduction, revealed that increased root hair density alone does not result in hypersusceptibility to $H$. schachtii. Therefore, we conclude that ethylene-mediated root hair development, per se, is not the cause of the altered susceptibilities of the rhdl alleles, the ACC- and AVG-treated seedlings, nor the tested ethylene mutants. Rather, it appears that ethylene acts as a positive regulator of root hair development and susceptibility to $H$. schachtii in A. thaliana.

We determined that the hypersusceptibilities of rhdl-4 and of the ethylene-overproducing mutant eto 3 could be attributed, at least in part, to an increased attraction of infective nematode juveniles to the roots of these plants. The observation that $H$. schachtii attraction is influenced by the ethylene signal transduction pathway could indicate that, through coevolution with host plants, the nematode has developed a response behavior to a (by)product of an important and ubiquitous plant signal transduction pathway. Furthermore, when considering that a nematode attack elicits elevated levels of endogenous ethylene in plant roots (Glazer et al. 1983; Glazer et al. 1985; Volkmar 1991), one could envision a feedback loop leading to increased attractiveness and, therefore, increased susceptibility of host roots under attack. Responsiveness to a (by)product of the ethylene signal transduction pathway would thereby confer an evolutionary advantage to the nematode.

Our finding that ethylene plays a role in the reproductive success of cyst nematodes agrees with recent observations that the phytohormone auxin is required for the formation of syncytia (Goverse et al. 2000). In addition to demonstrating the importance of auxin in syncytium development, Goverse et al. (2000) observed that eto1, eto2, and eto3 show hypersusceptibility to $H$. schachtii and that these hypersusceptibilities are indicative of their relative levels of ethylene overproduction, e.g., eto3 is the strongest eto mutation and was observed to host the greatest number of female nematodes. Although our data do not reflect such a relationship among the eto mutants, our data do clearly show a hypersusceptibility of the eto mutants to $H$. schachtii. Research by Goverse et al. (2000) has shown that a lack of auxin-inducible ethylene production in the form of the axr2 mutant hinders successful cyst nematode parasitism. Additionally, we have shown here that inhibited ethylene signal transduction, either through genetic lesions (etr1-1, ein2-1, and ein3-1) or through an applied chemical (AVG), inhibits plant susceptibility to a cyst nematode. Whereas Goverse et al. (2000) showed changes in the morphology of syncytia in ethylene mutants, our results add increased root attraction to the growing list of ethylene effects on A. thaliana susceptibility to $H$. schachtii.

rhd1-1 was found in a screen designed to identify $A$. thaliana mutants altered in root hair development. Epistasis analysis between rhd1-1 and other root hair defective mutants ( $r h d 2, r h d 3$, and $r h d 4$ ) indicated that the RHD1 protein acts early in root hair formation (Schiefelbein and Somerville 1990). Our finding that rhdl-4 likely is altered in its ethylene response supports the notion that RHDl is a regulator of root hair development because ethylene has been shown to promote root hair development in A. thaliana (Tanimoto et al.

Table 3. The attraction of Heterodera schachtii second-stage juveniles (J2) to wild-type Arabidopsis thaliana ecotype Columbia (Col-0), rhd1-4, and eto 3 root exudates at $20 \mathrm{~h}$ after $\mathrm{J} 2$ placement, equidistant to root exudates tested in pairwise combinations

\begin{tabular}{|c|c|c|c|c|c|c|c|c|}
\hline & Col-0 & versus & Control & rhd1-4 & versus & Col-0 & versus & Col-0 \\
\hline $\begin{array}{l}\text { Total }{ }^{\mathrm{a}} \\
\text { Mean }^{\mathrm{b}}\end{array}$ & $\begin{array}{c}25 \\
2.1 \pm 0.3^{c}\end{array}$ & & $\begin{array}{c}14 \\
1.2 \pm 0.3\end{array}$ & $\begin{array}{c}49 \\
4.1 \pm 0.8^{\mathrm{c}}\end{array}$ & & $\begin{array}{c}21 \\
1.8 \pm 0.5\end{array}$ & $\begin{array}{c}112 \\
9.3 \pm 2.4^{\mathrm{c}}\end{array}$ & $\begin{array}{c}51 \\
4.3 \pm 1.3\end{array}$ \\
\hline
\end{tabular}

a Total $\mathrm{J} 2$ attracted over 12 replications. Data are from one representative experiment.

${ }^{\mathrm{b}}$ Mean number of $\mathrm{J} 2$ attracted per replication \pm the standard error.

${ }^{\mathrm{c}}$ Denotes significant difference $(P<0.05)$ between the means of attracted $\mathrm{J} 2$ for each pairwise comparison as determined by one-tailed paired Student's $t$ test. 
1995). Whereas our data suggest that RHDI directly or indirectly mediates the ethylene signal transduction pathway, how this process occurs is unknown. Epistasis analyses between rhdl-4 and known hormone signal transduction mutants are currently underway in order to elucidate the position of RHD1 relative to genes known to reside in the ethylene signal transduction pathway.

The nature of the rhdl-4 root epidermal cell deformation phenotype is not resolved at this time. The observation that AVG suppresses this phenotype in rhdl-4 suggests that the deformation is ethylene dependent. The observations that $\mathrm{AgNO}_{3}$ is not able to suppress the epidermal cell deformation in rhdl-4 and that ACC is not able to induce the epidermal cell deformation phenotype in wild-type plants, however, contradict the AVG experiments and suggest that the epidermal cell deformation phenotype is ethylene independent. One possibility is that AVG also affects a pathway that leads to epidermal cell expansion. This conclusion is supported by the observation that none of the ethylene mutants had an epidermal cell deformation phenotype that we could observe. Also, because ACC-treated Col-0 and ethylene-overproducing mutants are hypersusceptible in the absence of epidermal cell deformation, we conclude that the epidermal cell deformation is without influence on susceptibility.

In the studies described here, we present an example of a plant signal transduction pathway that is directly involved in mediating susceptibility to the cyst nematode $H$. schachtii. We also presented a strong case that RHD1 is involved in ethylene signaling. Further characterization of this association is not only of academic interest but also of practical relevance because understanding this compatible interaction may open avenues to generate plants that are less susceptible to cyst nematodes.

\section{MATERIALS AND METHODS}

\section{Plant and nematode materials and inoculations.}

Second-generation progeny (M2) of ethyl methanesulfonate (EMS)-mutated (Lehle Seeds, Round Rock, TX, U.SA.) and wild-type $A$. thaliana seeds, both Col-0 ecotype, were used in the mutant screen that identified rhdl-4. The screen itself is presented elsewhere (Baum et al. 2000). All A. thaliana plants were grown from surface-sterilized seeds on modified Knop medium solidified with $0.8 \%$ Daishin agar (Brunschwig Chemie, Amsterdam, The Netherlands) (Sijmons et al. 1991) in 9-cm petri dishes (Fisher Scientific, Pittsburgh, PA, U.S.A.) or Falcon 12-well plates (Becton Dickinson, Lincoln Park, NJ, U.S.A.), which were sealed with Parafilm (American National Can, Menasha, WI, U.S.A.) and maintained at $26^{\circ} \mathrm{C}$ on a cycle of $12 \mathrm{~h}$ day-12 h night. A $H$. schachtii field population, designated TN101 (provided by G. Tylka, Iowa State University, and obtained originally from T. Niblack, University of Missouri), was grown in greenhouse cultures on cabbage or sugar beet plants. This nematode culture had been propagated previously for 10 years on cabbage and can be considered inbred by mass selection. Eggs were isolated, and J2 were hatched and surface sterilized, as described in Baum et al. (2000). Ten- to twelve-day-old $A$. thaliana plants were inoculated with approximately 300 surface-sterilized $\mathbf{J} 2$ that were suspended in sterile, $1.5 \%$ low-melting-point agarose (GIBCO-BRL, Grand Island, NY, U.S.A.) at $37^{\circ} \mathrm{C}$. A. thaliana ethylene and root hair mutants were obtained from the Arabidopsis Biological Resource Center at The Ohio State University.

Assessment of the ethylene mutants was performed over three experiments. The experimental error of experiments 1 and 2 was determined by $F$ statistic to not differ significantly. These data were, therefore, combined to increase sample size (i.e., mutant and Col-0 observations were 11 to 21 , except for eto 2 and eto3, which had four and six observations, respectively, as a result of their absence in experiment 1 ). In experiment 3 , mutant and Col-0 observations were 16 to 28 plants per line. The means and standard error of the means were determined by least square means analysis to account for unequal observation numbers between the mutants and wild-type Col-0. Significance was determined by least significant difference analysis $(P<0.05)$. One inoculation experiment for the $t t g$ and $g l 2$ mutants was performed, comprising 25 to 28 total plants tested for each mutant. Significance was determined by paired Student's $t$ test analysis $(P<0.05)$. To assess the ethylene mutants and $t t g$ and $g l 2$, tested plants were arranged arbitrarily with wild-type controls in 12-well culture plates and assessed for sedentary J2 at $7 \mathrm{dpi}$ and $\mathrm{J} 4$ females at $15 \mathrm{dpi}$ with a dissecting microscope.

\section{Root measurements.}

Plants were allowed to germinate and grow for 3 days on horizontally placed petri dishes containing growth medium. All plates were then tilted to approximately $60^{\circ}$ to promote unidirectional growth of all plant roots. All measurements were performed 10 days after germination. Root length (distance from the crown to the tip of the main root) and root hair length were measured with a reticle installed in an eyepiece of a dissecting microscope. Fifteen plants for each tested $A$. thaliana line were measured for root length. Root hairs were chosen arbitrarily from regions of roots that had fully formed root hairs. Twenty root hairs were measured from each of seven plants per $A$. thaliana line tested. Root-hair densities were determined by counting the root hairs in 1-mm root segments that exhibited uniform root hair stands. Twenty-five of the 1-mm segments were measured from 15 total plants for each $A$. thaliana line tested.

\section{Ethylene inhibitors and precursor tests.}

AVG (Sigma, St. Louis, MO, U.S.A.), $\mathrm{AgNO}_{3}$ (Fisher Scientific), and ACC (Sigma) stock solutions were made in distilled water, then filter sterilized through $0.2-\mu \mathrm{m}$ pore filters (Nalge Nunc International, Rochester, NY, U.S.A.). For the inoculation experiments, $50 \mu \mathrm{l}$ of appropriately diluted solutions were added to the surface of individual wells of 12-well plates ( $1 \mathrm{ml}$ of Knop medium per well) and allowed to absorb for 1 day before planting. Plants were inoculated and assessed as described above. Ten to twelve plants were assessed for each $A$. thaliana line tested. Significant differences were determined by paired Student's $t$ test $(P<0.05)$. The effect of AVG on J2 viability was determined by incubating freshly hatched $\mathrm{J} 2$ in sterile culture tubes with water containing either 0,10 , or $100 \mu \mathrm{M}$ AVG. Four tubes per treatment were prepared. Tubes were incubated at $26^{\circ} \mathrm{C}$ for $3 \mathrm{~h}$, after which an aliquot of the tube solution was spread on a microscope slide. A dissecting microscope was used to determine the percent of live $\mathbf{J} 2$ by counting moving worms and dividing by the total number of worms present. An identical count was conducted after a 26-h incubation. There were no differences in the per- 
cent of live $\mathrm{J} 2$ between the AVG solutions and the water control at both observations. Significance was analyzed with a two-sample Student's $t$ test $(P<0.05)$.

The evaluation of wild-type and rhdl-4 root morphology resulting from growth on AVG-, $\mathrm{AgNO}_{3^{-}}$, or ACC-supplemented medium was accomplished by first incorporating appropriate amounts of each compound into precooled Knop medium to obtain the desired final concentration. Petri plates were then poured, and seeds of each A. thaliana line were planted as described above. Root and root hair lengths and densities were determined as described above.

\section{Attraction assays.}

Seeds of wild-type Col-0, rhd1-4, and etol-1 were planted and allowed to grow for 10 days in $9-\mathrm{cm}$ petri dishes, as described above. After this growth period, plants were carefully removed. A cork borer was used to remove approximately 7mm-diameter growth medium plugs from areas that had been immediately adjacent to roots and, therefore, contained root exudates. Medium plugs were transferred in pairwise combinations, as described above, to 6-cm petri dishes (Fisher Scientific) containing Knop medium. Transferred plugs were spaced $3.5 \mathrm{~cm}$ apart from edge to edge, and $1.5 \%$ low-melting-point agarose was applied to seal the plug-growth-medium boundaries and displace trapped air. Approximately 50 surface-sterilized $\mathrm{J} 2 \mathrm{~s}$ in a drop of low-melting-point agarose were added to the center of the plates within equal distance to the two plugs. Nematodes that were attracted into either plug were counted after $20 \mathrm{~h}$ in the dark at room temperature. Twelve replications were tested for each combination.

\section{ACKNOWLEDGMENTS}

This is journal paper J-18400 of the Iowa Agriculture and Home Economics Experiment Station, Ames, Iowa, project 3502. This work was supported by the Hatch Act and State of Iowa funds. We gratefully acknowledge funding by the Iowa Soybean Promotion Board, the Iowa State University Biotechnology Council and Carver Trust Fund, and USDA-NRICGP awards 97-35302-4866 and 99-35302-7938. We thank $\mathrm{T}$. Baskin for kindly providing the rebl mutant lines. We also thank T. R. Maier for skillful technical assistance and C. Bronson and G. L. Tylka, Iowa State University, for critical review of the manuscript. Finally, we thank T. Baskin, J. Dangl, J. Schiefelbein, and G. Seifert for helpful comments and discussion.

\section{LITERATURE CITED}

Baskin, T. I., Betzner, A. S., Hoggart, R., Cork, A., and Williamson, R. E. 1992. Root morphology mutants in Arabidopsis thaliana. Aust. J. Plant Physiol. 19:427-437.

Baum, T. J., Wubben, M. J. E., II, Hardy, K. A., Su, H., and Rodermel, S. R. 2000. A screen for Arabidopsis thaliana mutants with altered susceptibility to Heterodera schachtii. J. Nematol. 32:166-173.

Beyer, E. M., Jr. 1976. A potent inhibitor of ethylene action in plants. Plant Physiol. 58:268-271.

Beyer, E. M., Jr. 1979. Effect of silver ion, carbon dioxide, and oxygen on ethylene action and metabolism. Plant Physiol. 63:169-173.

Bleecker, A. B., Estelle, M. A., and Somerville, C. R. 1988. Insensitivity to ethylene conferred by a dominant mutation in Arabidopsis thaliana. Science 241:1086-1089.

Cao, X. F., Linstead, P., Berger, F., Kieber, J., and Dolan, L. 1999. Differential ethylene sensitivity of epidermal cells is involved in the establishment of cell pattern in the Arabidopsis root. Physiol. Plant. 106:311-317.

Davis, E. L., Hussey, R. S., Baum, T. J., Bakker, J., Schots, A., Rosso, M.-N., and Abad, P. 2000. Nematode parasitism genes. Annu. Rev.
Phytopathol. 38:365-396.

De Boer, J. M., Yan, Y., Wang, X., Smant, G., Hussey, R. S., Davis, E. L., and Baum, T. J. 1999. Developmental expression of secretory $\beta$ 1,4-endoglucanases in the subventral esophageal glands of Heterodera glycines. Mol. Plant-Microbe Interact. 12:663-669.

Eliasson, L., and Bollmark, M. 1988. Ethylene as a possible mediator of light-induced inhibition of root growth. Physiol. Plant 72:605-609.

Glazer, I., Orion, D., and Apelbaum, A. 1983. Interrelationships between ethylene production, gall formation, and root-knot nematode development in tomato plants infected with Meloidogyne javanica. J. Nematol. $15: 539-544$.

Glazer, I., Apelbaum, A., and Orion, D. 1985. Effect of inhibitors and stimulators of ethylene production on gall development in Meloidogyne javanica-infected tomato roots. J. Nematol. 17:145-149.

Goverse, A., Overmars, H., Engelbertink, J., Schots, A., Bakker, J., and Helder, J. 2000. Both induction and morphogenesis of cyst nematode feeding cells are mediated by auxin. Mol. Plant-Microbe Interact. 13:1121-1129.

Guzman, P., and Ecker, J. R. 1990. Exploiting the triple response of Arabidopsis to identify ethylene-related mutants. Plant Cell 2:513-523.

Jones, M. G. K. 1981. Host cell responses to endoparasitic nematode attack: Structure and function of giant cells and syncytia. Ann. Appl. Biol. 97:353-372.

Kieber, J. J., and Ecker, J. R. 1993. Ethylene gas: It's not just for ripening any more. Trends Genet. 9:356-362.

Kieber, J. J., Rothenberg, M., Roman, G., Feldmann, K. A., and Ecker, J. R. 1993. CTR1, a negative regulator of the ethylene response pathway in Arabidopsis, encodes a member of the Raf family of protein kinase. Cell 72:427-441.

Masucci, J. D., and Schiefelbein, J. W. 1996. Hormones act downstream of $T T G$ and $G L 2$ to promote root hair outgrowth during epidermis development in the Arabidopsis root. Plant Cell 8:1505-1517.

Pitts, R. J., Cernac, A., and Estelle, M. 1998. Auxin and ethylene promote root hair elongation in Arabidopsis. Plant J. 16:553-560.

Rodriguez, F. I., Esch, J. J., Hall, A. E., Binder, B. M., Schaller, G. E., and Bleecker, A. B. 1999. A copper cofactor for the ethylene receptor ETR1 from Arabidopsis. Science 283:996-998.

Roman, G., Lubarsky, B., Kieber, J. J., Rothenberg, M., and Ecker, J. R. 1995. Genetic analysis of ethylene signal transduction in Arabidopsis thaliana: Five novel mutant loci integrated into a stress response pathway. Genetics 139:1393-1409.

Schiefelbein, J. W., and Somerville, C. 1990. Genetic control of root hair development in Arabidopsis thaliana. Plant Cell 2:235-243.

Schneider, K., Wells, B., Dolan, L., and Roberts, K. 1997. Structural and genetic analysis of epidermal cell differentiation in Arabidopsis primary roots. Development 124:1789-1798.

Sijmons, P. C., Grundler, F. M. W., Von Mende, N., Burrows, P. R., and Wyss, U. 1991. Arabidopsis thaliana as a new model host for plantparasitic nematodes. Plant J. 1:245-254.

Smant, G., Stokkermans, J. P. W. G., Yang, Y., De Boer, J. M., Baum, T. J., Wang, X., Hussey, R. S., Gommers, F. J., Henrissat, B., Davis, E. L., Helder, J., Schots, A., and Bakker, J. 1998. Endogenous cellulases in animals: Isolation of $\beta$-1,4-endoglucanases from two species of plant-parasitic nematodes. Proc. Natl. Acad. Sci. USA 95:4906-4911.

Tanimoto, M., Roberts, K., and Dolan, L. 1995. Ethylene is a positive regulator of root hair development in Arabidopsis thaliana. Plant J. 8:943-948.

Volkmar, K. M. 1991. Abscisic acid and ethylene increase in Heterodera avenae-infected tolerant or intolerant oat cultivars. J. Nematol. 23:425-431.

Wang, X., Meyers, D., Yan, Y., Baum, T., Smant, G., Hussey, R., and Davis, E. 1999. In planta localization of a $\beta$-1,4-endoglucanase secreted by Heterodera glycines. Mol. Plant Microbe Interact. 12:64-67.

Wilson, A. K., Pickett, F. B., Turner, J. C., and Estelle, M. 1990. A dominant mutation in Arabidopsis confers resistance to auxin, ethylene and abscisic acid. Mol. Gen. Genet. 222:377-383.

Wyss, U. 1992. Observations on the parasitic behavior of Heterodera schachtii throughout its development inside roots. Fundam. Appl. Nematol. 15:75-89

Wyss, U., and Grundler, F. M. W. 1992. Feeding behavior of sedentary plant-parasitic nematodes. Neth. J. Plant Pathol. 2S:165-173.

Wyss, U., and Zunke, U. 1986. Observation on the behavior of second stage juveniles of Heterodera schachtii inside host roots. Rev. Nematol. 9(2):153-165.

Yang, S. F., and Hoffman, N. E. 1984. Ethylene biosynthesis and its regulation in higher plants. Annu. Rev. Plant Physiol. 35:155-189. 